\title{
Fokker-Planck equation for Kolmogorov operators associated to stochastic PDE with multiplicative noise
}

\section{Yu Shi and Bin Liu*}

\section{"Correspondence:}

binliu@mail.hust.edu.cn

School of Mathematics and

Statistics, Huazhong University of

Science and Technology, Wuhan,

Hubei 430074, P.R. China

\begin{abstract}
In this paper, we show that the Kolmogorov operator $K_{0}$ associated to a stochastic PDE with multiplicative noise can be extended to the infinitesimal generator $(K, D(K))$ of the corresponding transition semigroup $\left\{P_{t}\right\}_{t \geq 0}$ in a proper weighted space. Then we apply the result to obtain the existence and uniqueness of a solution for the Fokker-Planck equation involving the Kolmogorov operator $K_{0}$.

MSC: 60H15; 35R15; 47D07
\end{abstract}

Keywords: Kolmogorov operator; stochastic PDE; transition semigroup; infinitesimal generator; Fokker-Planck equation

\section{Introduction}

Let $H$ be a separable Hilbert space (with norm $|\cdot|$ and inner product $\langle\cdot, \cdot\rangle)$ ), and $L(H, H)$ be the Banach space of all bounded linear operators from $H$ to $H$. We consider the stochastic differential equation in $H$

$$
\left\{\begin{array}{l}
d X(t)=[A X(t)+F(X(t))] d t+G(X(t)) d W(t), \quad t \geq 0 \\
X(0)=x \in H
\end{array}\right.
$$

where $A: D(A) \subset H \rightarrow H$ is the infinitesimal generator of a strongly continuous semigroup $\left(e^{t A}\right)_{t \geq 0}$ in $H, F: D(F) \subset H \rightarrow H$, and $G: H \rightarrow L(H, H)$ are measurable mappings, and $(W(t))_{t \geq 0}$ is a cylindrical Wiener process on $H$, defined on a filtered probability space $(\Omega, \mathcal{F}, \mathbb{P})$, and adapted to some filtration $\left(\mathcal{F}_{t}\right)_{t \geq 0}$ that is assumed to be right-continuous and complete in the sense that $\left(\mathcal{F}_{0}\right)$ contains all $P$-null sets. The precise assumptions on $F$ and $G$ are given in Hypothesis 2.1 below.

It is well known that under Hypothesis 2.1, (1.1) has a unique mild solution $(X(t, x))_{t \geq 0, x \in H}$ (see, for instance, $[1,2])$, that is, for any $x \in H$ the process $(X(t, x))_{t \geq 0}$ is adapted to the filtration $\left(\mathcal{F}_{t}\right)_{t \geq 0}$, and it is continuous in mean square and fulfills the integral equation

$$
X(t, x)=e^{t A} x+\int_{0}^{t} e^{(t-s) A} F(X(s, x)) d s+\int_{0}^{t} e^{(t-s) A} G(X(s, x)) d W(s)
$$


for $\mathbb{P}$-a.e. $\omega \in \Omega$ and any $t \geq 0$. Moreover, a straightforward computation shows that for any $T>0$ there exists $c>0$ such that

$$
\sup _{t \in[0, T]}|X(t, x)-X(t, y)| \leq c|x-y|, \quad \forall x, y \in H
$$

and

$$
\sup _{t \in[0, T]} \mathbb{E}|X(t, x)| \leq c(1+|x|), \quad \forall x \in H
$$

where the expectation is taken with respect to $\mathbb{P}$. As we shall see in Lemma 2.4, estimates (1.3) and (1.4) allow us to define the transition semigroup $\left\{P_{t}\right\}_{t \geq 0}$ associated to (1.2) in the space $C_{b, 1}(H)$ (see below for a precise definition), by the setting

$$
P_{t} \varphi(x)=\mathbb{E}[\varphi(X(t, x))], \quad \varphi \in C_{b, 1}(H), t \geq 0, x \in H .
$$

It is not hard to prove that $\left\{P_{t}\right\}_{t \geq 0}$ maps $C_{b, 1}(H)$ into $C_{b, 1}(H)$ and satisfies the semigroup property, see [3], but it is not a strongly continuous semigroup. However, we can define the infinitesimal generator $(K, D(K))$ of $\left\{P_{t}\right\}_{t \geq 0}$ in $C_{b, 1}(H)$ as

$$
\left\{\begin{aligned}
D(K)= & \left\{\varphi \in C_{b, 1}(H): \exists \psi \in C_{b, 1}(H), \lim _{t \rightarrow 0^{+}} \frac{P_{t} \varphi(x)-\varphi(x)}{t}=\psi(x), x \in H,\right. \\
& \left.\sup _{t \in(0,1)}\left\|\frac{P_{t} \varphi(x)-\varphi(x)}{t}\right\|_{0,1}<\infty\right\}, \\
K \varphi(x)= & \lim _{t \rightarrow 0^{+}} \frac{P_{t} \varphi(x)-\varphi(x)}{t}, \quad \varphi \in D(K), x \in H .
\end{aligned}\right.
$$

Naturally, we are interested in the connections between $(K, D(K))$ and the Kolmogorov operator

$$
K_{0} \varphi(x)=\frac{1}{2} \operatorname{Tr}\left[G(x) G(x)^{*} D^{2} \varphi(x)\right]+\left\langle x, A^{*} D \varphi(x)\right\rangle+\langle D \varphi(x), F(x)\rangle, \quad x \in H
$$

where $\varphi: H \rightarrow \mathbb{R}$ is a suitable function and Tr means trace.

In this paper, we will study the relationship between Kolmogorov operator $K_{0}$ and the infinitesimal generator $(K, D(K))$ of the transition semigroup $\left\{P_{t}\right\}_{t \geq 0}$ and solve the FokkerPlanck equation corresponding to $K_{0}$ (i.e. the dual of the Kolmogorov equation). As the first main result (see Theorem 3.1 below), we show that $(K, D(K))$ extends the Kolmogorov operator $K_{0}$ defined on the domain $\mathscr{E}_{A}(H)$, which consists of the linear span of the real and imaginary parts of the functions

$$
x \mapsto e^{i\langle x, h\rangle}, \quad x \in H, h \in D\left(A^{*}\right),
$$

and the set $\mathscr{E}_{A}(H)$ is a $\pi$-core (cf. Section 2) for $(K, D(K))$.

Let $\mathcal{M}(H)$ be the space of all finite Borel measures on $H, \mathcal{M}_{k}(H)$ be the set of all $\mu \in \mathcal{M}(H)$ such that $\int_{H}|x|^{k}|\mu|(d x)<\infty$, where $|\mu|$ is the total variation of $\mu$, the second main result is that for any $\mu \in \mathcal{M}_{1}(H)$, there exist a family of measures $\left\{\mu_{t}\right\}_{t \geq 0} \subset \mathcal{M}_{1}(H)$ fulfilling

$$
\int_{0}^{T} \int_{H}(1+|x|)\left|\mu_{t}\right|(d x) d t<\infty, \quad \forall T>0
$$


and the Fokker-Planck equation

$$
\left\{\begin{array}{l}
\frac{d}{d t} \int_{H} \varphi(x) \mu_{t}(d x)=\int_{H} K_{0} \varphi(x) \mu_{t}(d x), \quad t \geq 0, \varphi \in \mathscr{E}_{A}(H), \\
\mu_{0}=\mu \in \mathcal{M}_{1}(H) .
\end{array}\right.
$$

Moreover, this solution is given by $\left\{P_{t}^{*} \mu\right\}_{t \geq 0}$, where $\left\{P_{t}^{*}\right\}_{t \geq 0}$ is a semigroup defined by

$$
\left\langle\varphi, P_{t}^{*} f\right\rangle_{\sigma\left(C_{b, 1}(H), C_{b, 1}(H)^{*}\right)}=\left\langle P_{t} \varphi, f\right\rangle_{\sigma\left(C_{b, 1}(H), C_{b, 1}(H)^{*}\right)}
$$

where $\left(C_{b, 1}(H)\right)^{*}$ is the topological dual space of $C_{b, 1}(H)$; see Theorem 4.3.

For the problems with additive noise case (that is, $G$ is a constant in (1.1)), it is worth mentioning, the papers [3-5] have studied the problems by means of the OrnsteinUhlenbeck semigroup method. However, for the multiplicative noise case, the stochastic convolution in (1.2) is no longer a martingale, so this method and the Itô formula can not be used to a mild solution, thus new technology is needed. In particular, Da Prato and Zabczyk [6] studied the problem (1.1) by the semigroup method, based on the classical fixed point theorem, and used the factorization method to get an estimation of the stochastic convolution, which is a generalization of maximal inequality of martingales to stochastic convolution, and plays an important role in the following sections.

On the other hand, we remark that a great deal of research has been devoted to the extension of a differential operator like (1.6) to the infinitesimal generator of a diffusion semigroup in the space $L^{p}(H, v), p \geq 1$, where $v$ is an invariant measure for the semigroup (see, for example, [7-11] and references therein). In fact, if $v$ is an invariant measure for the semigroup (1.5), then the semigroup (1.5) can be extended to a strongly continuous contraction semigroup in $L^{p}(H, v), p \geq 1$.

Kolmogorov equations for measures in infinite dimensional space have been the object of many authors (see, e.g., [12-14] and references therein). For example, Bogachev and Röckner [14] considered the existence of measure valued solutions for the equation involving second order partial differential operators in infinite dimensional spaces. However, as an extension of the existing theory in [3], in this paper we pay attention to the existence and uniqueness of the solution of the Fokker-Planck equation for Kolmogorov operators associated to the SPDEs with multiplicative noise case which is important.

We organize the rest of this paper as follows. Some notation and preliminary results are in Section 2. In Section 3, we prove that $(K, D(K))$ extends the Kolmogorov operator $K_{0}$, and the set $\mathscr{E}_{A}(H)$ is a $\pi$-core for $(K, D(K))$. Finally, the existence and uniqueness of the solution for the Fokker-Planck equation (1.9) are given in Section 4; see Theorem 4.3.

\section{Preliminaries}

We list some notation which are applied in this paper. Let $H$ be a separable real Hilbert space (norm $|\cdot|$, inner product $\langle\cdot, \cdot\rangle)$, and $H^{*}$ represent its topological dual space. Let $\mathcal{M}(H)$ be the space of all finite Borel measures on $H$, and for any $k>0$, denote the set of all $v \in \mathcal{M}(H)$ such that $\int_{H}|x|^{k}|v|(d x)<\infty$ by $\mathcal{M}_{k}(H)$, where $|\nu|$ is the total variation of $v$. We denote the space of all linear bounded operators from $H$ into $H$ by $L(H)$, endowed with the norm

$$
\|T\|=\sup \{|T x| ; x \in H,|x|=1\}, \quad T \in L(H),
$$


and let $L_{2}(H)$ be the space of all Hilbert-Schmidt operators $L: H \rightarrow H$, endowed with the Hilbert-Schmidt norm. If $E$ is a Banach space (norm $|\cdot|)$, we denote $C_{b}(H ; E)$ as the linear space of all continuous and bounded mappings $\varphi: H \rightarrow E$, endowed with the norm

$$
\|\varphi\|_{0}=\sup _{x \in H}|\varphi(x)|
$$

is a Banach space. Moreover, $C_{b}^{1}(H ; E)$ represents the subspace of $C_{b}(H ; E)$ of all functions $\varphi: H \rightarrow E$ which are Fréchet differentiable on $H$ with a continuous and bounded derivative $D \varphi \in C_{b}(H ; L(H ; E))$, and the space $C_{b}^{k}(H ; E)$ for all $k \geq 2$ can be defined analogously. We shall write $C_{b}^{k}(H ; \mathbb{R})=C_{b}^{k}(H), k \in \mathbb{N}$ for short. For any $k>0$, let $C_{b, k}(H)$ be the space of all functions $\varphi: H \rightarrow \mathbb{R}$ such that the function $H \rightarrow \mathbb{R}, x \rightarrow\left(1+|x|^{k}\right)^{-1} \varphi(x)$ belongs to $C_{b}(H)$. The space $C_{b, k}(H)$ is a Banach space, endowed with the norm $\|\varphi\|_{0, k}=\left\|\left(1+|\cdot|^{k}\right)^{-1} \varphi\right\|_{0}$. In the following, we shall denote by $\left(C_{b, k}(H)\right)^{*}$ the topological dual space of $C_{b, k}(H)$.

If $\varphi \in C_{b}^{1}(H)$ and $x \in H$, we shall identify $D \varphi(x)$ with the unique element $h$ of $H$ such that

$$
D \varphi(x) y=\langle h, y\rangle, \quad x, y \in H .
$$

If $\varphi \in C_{b}^{2}(H)$ and $x \in H$, we shall identify $D^{2} \varphi(x)$ with the unique linear operator $T \in L(H)$ such that

$$
D^{2} \varphi(x)(y, z)=\langle T y, z\rangle, \quad x, y, z \in H .
$$

\section{Hypothesis 2.1}

$\left(\mathrm{H}_{0}\right) A: D(A) \subset H \rightarrow H$ is the infinitesimal generator of a strongly continuous semigroup $e^{t A}$ of type $\mathcal{G}(M, \omega)$, i.e. there exist $M>0$ and $\omega \in \mathbb{R}$ such that $\left\|e^{t A}\right\|_{L(H)} \leq M e^{\omega t}, t \geq 0$.

$\left(\mathrm{H}_{1}\right)$ Let $F: D(F) \subset H \rightarrow H$ be a continuous vector-field.

$\left(\mathrm{H}_{2}\right)$ Let $G: H \rightarrow L(H)$ be strongly continuous, and $e^{t A} G(x) \in L_{2}(H)$ for all $x \in H$.

$\left(\mathrm{H}_{3}\right)$ There exists $\kappa \in L_{\text {loc }}^{2}([0, \infty))$ such that

(i) $\left|e^{t A} F(x)\right|+\left\|e^{t A} G(x)\right\|_{L_{2}(H)} \leq \kappa(t)(1+|x|)$ for all $x \in H$,

(ii) $\left|e^{t A}(F(x)-F(y))\right|+\left\|e^{t A}(G(x)-G(y))\right\|_{L_{2}(H)} \leq \kappa(t)|x-y|$ for all $x, y \in H$.

The following result is about the existence and uniqueness of a mild solution for (1.1), the proof essentially follows by Theorem 1.7 in [2].

Proposition 2.2 Under Hypothesis 2.1, for any initial condition $x \in H$, problem (1.1) has a unique mild solution $(X(t, x))_{t \geq 0, x \in H}$, that is, for any $x \in H$, the process $(X(t, x))_{t \geq 0}$ is adapted to the filtration $\left(\mathcal{F}_{t}\right)_{t \geq 0}$, and it is continuous in mean square and fulfills the integral equation

$$
X(t, x)=e^{t A} x+\int_{0}^{t} e^{(t-s) A} F(X(s, x)) d s+\int_{0}^{t} e^{(t-s) A} G(X(s, x)) d W(s)
$$

for $\mathbb{P}$-a.e. $\omega \in \Omega$ and all $t>0, x \in H$. Moreover, for any $T>0, p \geq 2$, there exists $C_{T}>0$ such that

$$
\sup _{t \in[0, T]} \mathbb{E}|X(t, x)-X(t, y)| \leq C_{T}|x-y|, \quad \forall x, y \in H
$$


and

$$
\sup _{t \in[0, T]} \mathbb{E}\left[|X(t, x)|^{p}\right] \leq C_{T}\left(1+|x|^{p}\right), \quad \forall x \in H
$$

As the semigroups of operators which we will deal with are not strongly continuous, we introduce the notion of $\pi$-convergence in the space $C_{b}(H)$ (see [15]).

Definition 2.3 (i) A sequence $\left\{\varphi_{n}\right\}_{n \in \mathbb{N}} \subset C_{b}(H)$ is said to be $\pi$-convergent to a function $\varphi \in C_{b}(H)$ if for any $x \in H$ we have

$$
\lim _{n \rightarrow \infty} \varphi_{n}(x)=\varphi(x) \quad \text { and } \quad \sup _{n \in \mathbb{N}}\left\|\varphi_{n}\right\|_{0}<\infty
$$

Similarly, the $m$-indexed sequence $\left\{\varphi_{n_{1}, \ldots, n_{m}}\right\}_{n_{1}, \ldots, n_{m} \in \mathbb{N}} \subset C_{b}(H)$ is said to be $\pi$-convergent to $\varphi \in C_{b}(H)$ if for any $i \in\{1, \ldots, m-1\}$ there exists an $i$-indexed sequence $\left\{\varphi_{n_{1}, \ldots, n_{i}}\right\} \subset C_{b}(H)$, $n_{1}, \ldots, n_{i} \in \mathbb{N}$ such that

$$
\lim _{n_{i+1} \rightarrow \infty} \varphi_{n_{1}, \ldots, n_{i+1}} \stackrel{\pi}{=} \varphi_{n_{1}, \ldots, n_{i}}, \quad i \in\{1, \ldots, m-1\}
$$

and

$$
\lim _{n_{1} \rightarrow \infty} \varphi_{n_{1}} \stackrel{\pi}{=} \varphi
$$

We shall write

$$
\lim _{n_{1} \rightarrow \infty} \cdots \lim _{n_{m} \rightarrow \infty} \varphi_{n_{1}, \ldots, n_{m}} \stackrel{\pi}{=} \varphi
$$

or $\varphi_{n} \stackrel{\pi}{\rightarrow} \varphi$ as $n \rightarrow \infty$, when the sequence has one index.

(ii) For any subset $D \subset C_{b}(H)$ we say that $\varphi$ belongs to the $\pi$-closure of $D$, and we denote it by $\varphi \in \bar{D}^{\pi}$, if there exist $m \in \mathbb{N}$ and an $m$-indexed sequence $\left\{\varphi_{n_{1}, \ldots, n_{m}}\right\}_{n_{1}, \ldots, n_{m} \in \mathbb{N}} \subset D$ such that

$$
\lim _{n_{1} \rightarrow \infty} \cdots \lim _{n_{m} \rightarrow \infty} \varphi_{n_{1}, \ldots, n_{m}} \stackrel{\pi}{=} \varphi
$$

Finally, we shall say that a subset $D \subset C_{b}(H)$ is $\pi$-dense in $C \subset C_{b}(H)$ if $\bar{D}^{\pi}=C$.

Notice that since the convergence is pointwise we cannot take a diagonal sequence. However, in order to avoid heavy notations, we shall often assume that the sequence has one index.

As an extension of the $\pi$-convergent to the space $C_{b, k}(H)(k>0)$, a sequence $\left\{\varphi_{n}\right\}_{n \in \mathbb{N}} \subset$ $C_{b, k}(H)$ is said to be $\pi$-convergent to a function $\varphi \in C_{b, k}(H)$ if we have $\left(1+|x|^{k}\right)^{-1} \varphi_{n} \stackrel{\pi}{\rightarrow}$ $\left(1+|x|^{k}\right)^{-1} \varphi$ as $n \rightarrow \infty$ in $C_{b}(H)$. Similarly, we can define $\pi$-dense in the space $C_{b, k}(H)$ $(k>0)$.

Here we introduce some properties for the transition semigroup $\left\{P_{t}\right\}_{t \geq 0}$ in $C_{b, 1}(H)$, which can be proved by a similar argument to [3], and they play an important role in the proof of the results in the following sections. 
Lemma 2.4 Formula (1.5) defines a semigroup of operators $P_{t}, t \geq 0$ in $C_{b, 1}(H)$, and there exist a family of probability measures $\left\{\pi_{t}(x, \cdot), t \geq 0, x \in H\right\} \subset \mathcal{M}_{1}(H)$ and two constants $c_{0}>0, \omega_{0} \in \mathbb{R}$ such that

(i) $P_{t} \in L\left(C_{b, 1}(H)\right)$ and $\left\|P_{t}\right\|_{L\left(C_{b, 1}(H)\right)} \leq c_{0} e^{\omega_{0} t}$;

(ii) $P_{t} \varphi(x)=\int_{H} \varphi(y) \pi_{t}(x, d y)$, for any $t \geq 0, \varphi \in C_{b, 1}(H), x \in H$;

(iii) for any $\varphi \in C_{b, 1}(H), x \in H$, the function $\mathbb{R}^{+} \rightarrow \mathbb{R}, t \mapsto P_{t} \varphi(x)$ is continuous;

(iv) $P_{t} P_{s}=P_{t+s}$ for any $t, s \geq 0$ and $P_{0}=I$;

(v) for any $\varphi \in C_{b, 1}(H)$ and any sequence $\left(\varphi_{n}\right)_{n \in \mathbb{N}} \subset C_{b, 1}(H)$ such that $\frac{\varphi_{n}}{1+|\cdot|} \stackrel{\pi}{\rightarrow} \frac{\varphi}{1+|\cdot|}$ as $n \rightarrow \infty$, we have $\frac{P_{t} \varphi_{n}}{1+|\cdot|} \stackrel{\pi}{\rightarrow} \frac{P_{t} \varphi}{1+1 \cdot \mid}$ as $n \rightarrow \infty$, for any $t \geq 0$.

Lemma 2.5 Let $X(t, x)$ be the mild solution of problem (1.1) and let $P_{t}, t \geq 0$ be the associated transition semigroups in the space $C_{b, 1}(H)$ defined by (1.5). Let also $(K, D(K))$ be the associated infinitesimal generators, defined by (1.6). Then

(i) for any $\varphi \in D(K)$, we have $P_{t} \varphi \in D(K)$ and $K P_{t} \varphi=P_{t} K \varphi, t \geq 0$;

(ii) for any $\varphi \in D(K), x \in H$, the map $[0, \infty) \rightarrow \mathbb{R}, t \mapsto P_{t} \varphi(x)$ is continuously differentiable and $(d / d t) P_{t} \varphi(x)=P_{t} K \varphi(x)$;

(iii) given $c_{0} \geq 0$ and $\omega_{0}$ as in Lemma 2.4, for any $\lambda>\omega_{0}$ the linear operator $R(\lambda, K)$ on $C_{b, 1}(H)$ done by

$$
R(\lambda, K) f(x)=\int_{0}^{\infty} e^{-\lambda t} P_{t} f(x) d t, \quad f \in C_{b, 1}(H), x \in H,
$$

satisfies, for any $f \in C_{b, 1}(H)$

$$
\begin{aligned}
& R(\lambda, K) \in L\left(C_{b, 1}(H)\right), \quad\|R(\lambda, K)\|_{L\left(C_{b, 1}(H)\right)} \leq \frac{c_{0}}{\lambda-\omega_{0}}, \\
& R(\lambda, K) f \in D(K), \quad(\lambda I-K) R(\lambda, K) f=f .
\end{aligned}
$$

We call $R(\lambda, K)$ the resolvent of $K$ at $\lambda$.

Definition 2.6 We say that $D \subset D(K)$ is a $\pi$-core for the operator $(K, D(K))$, if $D$ is $\pi$-dense in $C_{b, 1}(H)$ and for any $\varphi \in D(K)$ there exist $m \in \mathbb{N}$ and an $m$-indexed sequence $\left\{\varphi_{n_{1}, \ldots, n_{m}}\right\}_{n_{1}, \ldots, n_{m} \in \mathbb{N}} \subset D$ such that

$$
\lim _{n_{1} \rightarrow \infty} \cdots \lim _{n_{m} \rightarrow \infty} \frac{\varphi_{n_{1}, \ldots, n_{m}}}{1+|\cdot|} \stackrel{\pi}{=} \frac{\varphi}{1+|\cdot|}
$$

and

$$
\lim _{n_{1} \rightarrow \infty} \ldots \lim _{n_{m} \rightarrow \infty} \frac{K \varphi_{n_{1}, \ldots, n_{m}}}{1+|\cdot|} \stackrel{\pi}{=} \frac{K \varphi}{1+|\cdot|} .
$$

\section{A core for operator $K$}

In this section, we give the first main result which is a better understanding of the relationships between the infinitesimal generator $K$ and the Kolmogorov differential operator $K_{0}$ defined by (1.7).

Theorem 3.1 Assume, besides Hypothesis 2.1, that there exist two constants $M_{1}>0$ and $\omega_{1} \in \mathbb{R}$ such that the function $\kappa$ in hypothesis $\left(\mathrm{H}_{3}\right)$ satisfies $\kappa(t) \leq M_{1} e^{\omega_{1} t}, t \geq 0$, then the 
operator $(K, D(K))$ is an extension of $K_{0}$, that is, for any $\varphi \in \mathscr{E}_{A}(H)$, we have $\varphi \in D(K)$ and $K \varphi=K_{0} \varphi$. Moreover, $\mathscr{E}_{A}(H)$ is a $\pi$-core for $(K, D(K))$.

We split the proof in several steps. In Proposition 3.3 we will prove Theorem 3.1 in the case $F=0$, then Corollary 3.5 will show that $(K, D(K))$ is an extension of $K_{0}$ and $K \varphi=K_{0} \varphi$ for any $\varphi \in \mathscr{E}_{A}(H)$. Finally, Proposition 3.8 will complete the proof by the proper approximation sequence of $F$ and $G$.

Firstly, we need the following approximation result, proved in [5, Proposition 2.7].

Proposition 3.2 For any $\varphi \in C_{b}(H)$, there exist $m \in \mathbb{N}$ and an $m$-indexed sequence $\left\{\varphi_{n_{1}, \ldots, n_{m}}\right\}_{n_{1}, \ldots, n_{m} \in \mathbb{N}} \subset \mathscr{E}_{A}(H)$ such that

$$
\lim _{n_{1} \rightarrow \infty} \cdots \lim _{n_{m} \rightarrow \infty} \varphi_{n_{1}, \ldots, n_{m}} \stackrel{\pi}{=} \varphi
$$

Moreover, if $\varphi \in C_{b}^{1}(H)$, we can choose the sequence in such a way that (3.1) holds and

$$
\lim _{n_{1} \rightarrow \infty} \cdots \lim _{n_{m} \rightarrow \infty}\left\langle D \varphi_{n_{1}, \ldots, n_{m}}, h\right\rangle \stackrel{\pi}{=}\langle D \varphi, h\rangle, \quad \forall h \in H
$$

\subsection{The case $F=0$}

If $F=0$, we consider the transition semigroup associated to the stochastic differential equation in $H$

$$
\left\{\begin{array}{l}
d Z(t)=A Z(t) d t+G(Z(t)) d W(t), \quad t \geq 0 \\
Z(0)=x \in H
\end{array}\right.
$$

We recall that the mild solution is given by the process

$$
Z(t, x)=e^{t A} x+W_{A}(t)
$$

where $W_{A}(t)=\int_{0}^{t} e^{(t-s) A} G(Z(s, x)) d W(s)$, and the semigroup $\left\{R_{t}\right\}_{t \geq 0}$ is defined by setting

$$
R_{t} \varphi(x)=\mathbb{E}[\varphi(Z(t, x))], \quad \varphi \in C_{b, 1}(H), t \geq 0, x \in H
$$

We define the infinitesimal generator $L: D(L) \rightarrow C_{b, 1}(H)$ of the semigroup $\left\{R_{t}\right\}_{t \geq 0}$ in $C_{b, 1}(H)$ as in (1.6), with $L$ replacing by $K$ and $R_{t}$ replacing $P_{t}$.

Proposition 3.3 Assuming that the conditions of Theorem 3.1 hold, for any $\varphi \in \mathscr{E}_{A}(H)$ we have $\varphi \in D(L)$ and

$$
L \varphi(x)=\frac{1}{2} \operatorname{Tr}\left[G(x) G(x)^{*} D^{2} \varphi(x)\right]+\left\langle x, A^{*} D \varphi(x)\right\rangle, \quad x \in H .
$$

The set $\mathscr{E}_{A}(H)$ is a $\pi$-core for $(L, D(L))$, and for any $\varphi \in D(L) \cap C_{b}^{1}(H)$ there exist $m \in \mathbb{N}$ and an $m$-indexed sequence $\left\{\varphi_{n_{1}, \ldots, n_{m}}\right\}_{n_{1} \in \mathbb{N}, \ldots, n_{m} \in \mathbb{N}} \subset \mathscr{E}_{A}(H)$ such that

$$
\lim _{n_{1} \rightarrow \infty} \cdots \lim _{n_{m} \rightarrow \infty} \frac{\varphi_{n_{1}, \ldots, n_{m}}}{1+|\cdot|} \stackrel{\pi}{=} \frac{\varphi}{1+|\cdot|}
$$


and

$$
\lim _{n_{1} \rightarrow \infty} \ldots \lim _{n_{m} \rightarrow \infty} \frac{\frac{1}{2} \operatorname{Tr}\left[G(\cdot) G(\cdot)^{*} D^{2} \varphi_{n_{1}, \ldots, n_{m}}\right]+\left\langle\cdot, A^{*} D \varphi_{n_{1}, \ldots, n_{m}}\right\rangle}{1+|\cdot|} \stackrel{\pi}{=} \frac{L \varphi}{1+|\cdot|} .
$$

Moreover, if $\varphi \in D(L) \cap C_{b}^{1}(H)$ we can choose the sequence in such a way that (3.6), (3.7) hold and

$$
\lim _{n_{1} \rightarrow \infty} \cdots \lim _{n_{m} \rightarrow \infty}\left\langle D \varphi_{n_{1}, \ldots, n_{m}}, h\right\rangle \stackrel{\pi}{=}\langle D \varphi, h\rangle
$$

for any $h \in H$.

Proof By the mean value theorem, for any $\varphi \in \mathscr{E}_{A}(H)$

$$
\varphi\left(e^{t A} x+y\right)=\varphi\left(e^{t A} x\right)+\left\langle D_{x} \varphi\left(e^{t A} x\right), y\right\rangle+\frac{1}{2}\left\langle D_{x}^{2} \varphi\left(e^{t A} x+\sigma_{t}(y) y\right) y, y\right\rangle
$$

where $\sigma_{t}(y)$ is a Borel function from $H$ into $[0,1]$. Therefore

$$
\begin{aligned}
R_{t} \varphi(x) & =\mathbb{E}\left[\varphi\left(e^{t A} x+W_{A}(t)\right)\right] \\
& =\mathbb{E}\left[\varphi\left(e^{t A} x\right)+\frac{1}{2}\left\langle D_{x}^{2} \varphi\left(e^{t A} x+\sigma_{t}\left(W_{A}(t)\right) W_{A}(t)\right) W_{A}(t), W_{A}(t)\right\rangle\right],
\end{aligned}
$$

then

$$
\begin{aligned}
& \frac{R_{t} \varphi(x)-\varphi(x)}{t}-\frac{\varphi\left(e^{t A} x\right)-\varphi(x)}{t} \\
& \quad=\frac{1}{2} \mathbb{E}\left[\frac{1}{t}\left\langle D_{x}^{2} \varphi\left(e^{t A} x+\sigma_{t}\left(W_{A}(t)\right) W_{A}(t)\right) W_{A}(t), W_{A}(t)\right\rangle\right] .
\end{aligned}
$$

We first show that

$$
\frac{\varphi\left(e^{t A} x\right)-\varphi(x)}{t} \rightarrow\left\langle x, A^{*} D \varphi(x)\right\rangle, \quad \text { as } t \downarrow 0 .
$$

Again, by the mean value theorem,

$$
\varphi\left(e^{t A} x\right)-\varphi(x)=\left\langle D \varphi\left(x+\xi_{t} \cdot\left(e^{t A} x-x\right)\right), e^{t A} x-x\right\rangle,
$$

where $\xi_{t} \in[0,1]$. However, for arbitrary $x \in H$

$$
e^{t A} x-x=A \int_{0}^{t} e^{s A} x d s
$$

and

$$
\begin{aligned}
\frac{\varphi\left(e^{t A} x\right)-\varphi(x)}{t} & =\frac{1}{t}\left\langle D \varphi\left(x+\xi_{t} \cdot\left(e^{t A} x-x\right)\right), A \int_{0}^{t} e^{s A} x d s\right\rangle \\
& =\left\langle A^{*} D \varphi\left(x+\xi_{t} \cdot\left(e^{t A} x-x\right)\right), \frac{1}{t} \int_{0}^{t} e^{s A} x d s\right\rangle .
\end{aligned}
$$

Since $e^{t A} x-x \rightarrow 0, \frac{1}{t} \int_{0}^{t} e^{s A} x d s \rightarrow x$ as $t \rightarrow 0$, the relation (3.11) holds. 
- Assertion. For any $x \in H$, let $I(t)$ denote the right-hand side of (3.10), and set

$$
I^{\prime}(t)=\frac{1}{2} \mathbb{E}\left[\frac{1}{t}\left\langle D_{x}^{2} \varphi(x) W_{A}(t), W_{A}(t)\right\rangle\right]
$$

we have

$$
\lim _{t \rightarrow 0}\left|I(t)-I^{\prime}(t)\right|=0
$$

For any $x \in H$, set $y=W_{A}(t)=\int_{0}^{t} e^{(t-s) A} G(Z(s, x)) d W(s)$, then

$$
\begin{aligned}
\left|I(t)-I^{\prime}(t)\right| & =\frac{1}{2 t} \mathbb{E}\left[\left|\left\langle\left[D_{x}^{2} \varphi\left(e^{t A} x+\sigma_{t}(y) y\right)-D_{x}^{2} \varphi(x)\right] y, y\right\rangle\right|\right] \\
& \leq \frac{1}{2 t} \mathbb{E}\left[\left\|D_{x}^{2} \varphi\left(e^{t A} x+\sigma_{t}(y) y\right)-D_{x}^{2} \varphi(x)\right\| \cdot|y|^{2}\right] \\
& \leq \frac{1}{2 t}\left(\mathbb{E}\left[\left\|D_{x}^{2} \varphi\left(e^{t A} x+\sigma_{t}(y) y\right)-D_{x}^{2} \varphi(x)\right\|^{2}\right]\right)^{\frac{1}{2}} \cdot\left(\mathbb{E}\left[|y|^{4}\right]\right)^{\frac{1}{2}} .
\end{aligned}
$$

We shall use the following Burkholder estimate, see [6]; for a constant $c>0$, we have

$$
\mathbb{E}\left[\left|\int_{0}^{t} e^{(t-s) A} G(Z(s, x)) d W(s)\right|^{4}\right] \leq c \mathbb{E}\left[\left(\int_{0}^{t}\left\|e^{(t-s) A} G(Z(s, x))\right\|_{L_{2}(H)}^{2} d s\right)^{2}\right] .
$$

By the Minkowski inequality, on account of hypothesis $\left(\mathrm{H}_{3}\right)$ and (2.5), it renders

$$
\begin{aligned}
\frac{1}{t}\left(\mathbb{E}\left[|y|^{4}\right]\right)^{\frac{1}{2}} & \leq \frac{c}{t} \int_{0}^{t}\left(\mathbb{E}\left[\left\|e^{(t-s) A} G(Z(s, x))\right\|_{L_{2}(H)}^{4}\right]\right)^{\frac{1}{2}} d s \\
& \leq c_{1}\left(1+|x|^{4}\right)^{\frac{1}{2}} \frac{1}{t} \int_{0}^{t} \kappa(t-s)^{2} d s,
\end{aligned}
$$

and due to $\kappa(t) \leq M_{1} e^{\omega_{1} t}$, for any $T>0$, there exists a constant $c_{2}>0$ such that

$$
\frac{1}{t} \int_{0}^{t} \kappa(t-s)^{2} d s \leq \frac{M_{1}^{2}\left(e^{2 \omega_{1} t}-1\right)}{2 \omega_{1} t} \leq c_{2}, \quad t \in(0, T]
$$

Moreover, $\mathbb{E}\left[\left\|D_{x}^{2} \varphi\left(e^{t A} x+\sigma_{t}(y) y\right)-D_{x}^{2} \varphi(x)\right\|^{2}\right] \rightarrow 0$, as $t \downarrow 0$, therefore, taking into account (3.15), $\left|I(t)-I^{\prime}(t)\right| \rightarrow 0$, as $t \downarrow 0$ uniformly with respect to $x \in H$.

On the other side, from the Fubini theorem and the properties of the stochastic integral for cylindrical Winner processes (see, for instance, [6]), it follows that

$$
\mathbb{E}\left[\left\langle D_{x}^{2} \varphi(x) W_{A}(t), W_{A}(t)\right\rangle\right]=\mathbb{E}\left[\int_{0}^{t} \operatorname{Tr}\left[e^{(t-s) A} G(Z(s, x)) G(Z(s, x))^{*} e^{(t-s) A^{*}} D_{x}^{2} \varphi(x)\right] d s\right],
$$

then

$$
\begin{aligned}
I^{\prime}(t) & =\frac{1}{2} \mathbb{E}\left[\frac{1}{t} \int_{0}^{t} \operatorname{Tr}\left[e^{(t-s) A} G(Z(s, x)) G(Z(s, x))^{*} e^{(t-s) A^{*}} D_{x}^{2} \varphi(x)\right] d s\right] \\
& =\frac{1}{2} \mathbb{E}\left[\frac{1}{t} \int_{0}^{t} \sum_{k=1}^{\infty}\left\langle e^{(t-s) A} G(Z(s, x)) G(Z(s, x))^{*} e^{(t-s) A^{*}} D_{x}^{2} \varphi(x) e_{k}, e_{k}\right\rangle d s\right] \\
& =\frac{1}{2} \mathbb{E}\left[\sum_{k=1}^{\infty}\left\langle\frac{1}{t} \int_{0}^{t} e^{(t-s) A} G(Z(s, x)) G(Z(s, x))^{*} e^{(t-s) A^{*}} D_{x}^{2} \varphi(x) e_{k} d s, e_{k}\right\rangle\right]
\end{aligned}
$$


where the sequence $\left\{e_{k}\right\}_{k \in \mathbb{N}}$ is an orthonormal basis in $H$, by hypotheses $\left(\mathrm{H}_{2}\right),\left(\mathrm{H}_{3}\right)$, and (3.3), for any $x \in H$ it follows that

$$
\lim _{t \rightarrow 0} \frac{1}{t} \int_{0}^{t} e^{(t-s) A} G(Z(s, x)) G(Z(s, x))^{*} e^{(t-s) A^{*}} d s=G(x) G(x)^{*} .
$$

Therefore

$$
\begin{aligned}
\lim _{t \rightarrow 0} I^{\prime}(t) & =\frac{1}{2} \mathbb{E}\left[\sum_{k=1}^{\infty}\left\langle G(x) G(x)^{*} D_{x}^{2} \varphi(x) e_{k}, e_{k}\right\rangle\right] \\
& =\frac{1}{2} \operatorname{Tr}\left[G(x) G(x)^{*} D_{x}^{2} \varphi(x)\right]
\end{aligned}
$$

and

$$
\lim _{t \rightarrow 0} \frac{R_{t} \varphi(x)-\varphi(x)}{t}=\frac{1}{2} \operatorname{Tr}\left[G(x) G(x)^{*} D^{2} \varphi(x)\right]+\left\langle x, A^{*} D \varphi(x)\right\rangle .
$$

Moreover, in view of (3.10), we have

$$
\begin{aligned}
\left|\frac{R_{t} \varphi(x)-\varphi(x)}{t}\right| \leq & \left|\frac{\varphi\left(e^{t A} x\right)-\varphi(x)}{t}\right| \\
& +\frac{1}{2}\left|\mathbb{E}\left[\frac{1}{t}\left\langle D_{x}^{2} \varphi\left(e^{t A} x+\sigma_{t}\left(W_{A}(t)\right) W_{A}(t)\right) W_{A}(t), W_{A}(t)\right\rangle\right]\right| \\
\leq & \left|\left\langle A^{*} D \varphi\left(x+\xi_{t} \cdot\left(e^{t A} x-x\right)\right), \frac{1}{t} \int_{0}^{t} e^{s A} x d s\right\rangle\right| \\
& +\frac{1}{2}\left\|D^{2} \varphi\right\|_{0} \mathbb{E}\left[\frac{1}{t} \int_{0}^{t}\left\|e^{(t-s) A} G(Z(s, x))\right\|_{L_{2}(H)}^{2} d s\right],
\end{aligned}
$$

considering hypotheses $\left(\mathrm{H}_{0}\right),\left(\mathrm{H}_{3}\right)$, and (2.5), it yields

$$
\begin{aligned}
\left|\frac{R_{t} \varphi(x)-\varphi(x)}{t}\right| \leq & \left|\left\langle A^{*} D \varphi\left(x+\xi_{t} \cdot\left(e^{t A} x-x\right)\right), \frac{1}{t} \int_{0}^{t} e^{s A} x d s\right\rangle\right| \\
& +\frac{c_{2}}{2}\left\|D^{2} \varphi\right\|_{0}\left(1+|x|^{2}\right) \frac{1}{t} \int_{0}^{t} \kappa(t-s)^{2} d s .
\end{aligned}
$$

On account of (3.15) and $\lim _{t \rightarrow 0^{+}} \frac{1}{t} \int_{0}^{t} \kappa(t-s)^{2} d s \leq M_{1}^{2}$, this implies

$$
\sup _{t \in(0,1]} \frac{1}{t}\left\|R_{t} \varphi(x)-\varphi(x)\right\|_{0,1}<\infty
$$

thus, $\varphi \in D(L)$ and (3.5) holds.

Similar to the proof given in [5], now we prove that the set $\mathscr{E}_{A}(H)$ is a $\pi$-core for $(L, D(L))$. Let $\varphi \in D(L)$, for any $n_{2} \in \mathbb{N}$, set $\varphi_{n_{2}}(x)=n_{2} \varphi(x) /\left(n_{2}+|x|^{2}\right)$. Clearly, $\varphi_{n_{2}} \in C_{b}(H)$ and $(1+$ $|x|)^{-1} \varphi_{n_{2}} \stackrel{\pi}{\rightarrow}(1+|x|)^{-1} \varphi$ as $n_{2} \rightarrow \infty$. By Proposition 3.2, for any $n_{2} \in \mathbb{N}$ we fix a sequence $\varphi_{n_{2}, n_{3}} \subset \mathscr{E}_{A}(H)$ (for the sake of simplicity we assume that the sequence has only one index) such that $\varphi_{n_{2}, n_{3}} \stackrel{\pi}{\rightarrow} \varphi_{n_{2}}$ as $n_{3} \rightarrow \infty$. Set

$$
\varphi_{n_{1}, n_{2}, n_{3}, n_{4}}(x)=\frac{1}{n_{4}} \sum_{k=1}^{n_{4}} R_{\frac{k}{n_{1} n_{4}}} \varphi_{n_{2}, n_{3}}(x)
$$


for any $n_{1}, n_{2}, n_{3}, n_{4} \in \mathbb{N}$, so we can show that the sequence $\left(\varphi_{n_{1}, n_{2}, n_{3}, n_{4}}\right)$ fulfills (3.6) by a straightforward computation because of the continuity of the function $\mathbb{R}^{+} \rightarrow \mathbb{R}, t \rightarrow$ $R_{t} \varphi(x)$, for any $\varphi \in C_{b, 1}(H)$.

And likewise, for any $x \in H$ we have

$$
\begin{aligned}
& \lim _{n_{1} \rightarrow \infty} \ldots \lim _{n_{4} \rightarrow \infty} \frac{\frac{1}{2} \operatorname{Tr}\left[G(\cdot) G(\cdot)^{*} D^{2} \varphi_{n_{1}, \ldots, n_{4}}(x)\right]+\left\langle x, A^{*} D \varphi_{n_{1}, \ldots, n_{4}}(x)\right\rangle}{1+|x|} \\
& =\lim _{n_{1} \rightarrow \infty} \cdots \lim _{n_{4} \rightarrow \infty} \frac{L \varphi_{n_{1}, \ldots, n_{4}}(x)}{1+|x|} \\
& =\lim _{n_{1} \rightarrow \infty} \lim _{n_{2} \rightarrow \infty} \lim _{n_{3} \rightarrow \infty} \frac{n_{1} \int_{0}^{1 / n_{1}} L R_{t} \varphi_{n_{2}, n_{3}}(x) d t}{1+|x|} \\
& =\lim _{n_{1} \rightarrow \infty} \lim _{n_{2} \rightarrow \infty} \lim _{n_{3} \rightarrow \infty} \frac{n_{1}\left(R_{\frac{1}{n_{1}}} \varphi_{n_{2}, n_{3}}(x)-\varphi_{n_{2}, n_{3}}(x)\right)}{1+|x|} \\
& =\lim _{n_{1} \rightarrow \infty} \frac{n_{1}\left(R_{\frac{1}{n_{1}}} \varphi(x)-\varphi(x)\right)}{1+|x|}=\frac{L \varphi(x)}{1+|x|}
\end{aligned}
$$

where we have used the continuity of $t \mapsto L R_{t} \varphi_{n_{2}, n_{3}}(x)$ and the fact that $L R_{t} \varphi_{n_{2}, n_{3}}(x)=$ $(d / d t) R_{t} \varphi_{n_{2}, n_{3}}(x)$ (cf. Lemmas 2.4 and 2.5). In fact that any limit above is equibounded in $C_{b, 1}(H)$ with respect to the corresponding index by the construction of $\varphi_{n_{1}, n_{2}, n_{3}, n_{4}}(x)$, thus (3.7) holds.

If $\varphi \in D(L) \cap C_{b}^{1}(H)$, (3.8) can be proved by Proposition 3.2.

Theorem 3.4 Assume that the conditions of Theorem 3.1 hold, let $\left\{P_{t}\right\}_{t \geq 0}$ be the semigroup (1.5) and $\left\{R_{t}\right\}_{t \geq 0}$ be the semigroup (3.4), we denote by $(K, D(K)),(L, D(L))$ the corresponding infinitesimal generators in $C_{b, 1}(H)$. Then we have $D(L) \cap C_{b}^{1}(H)=D(K) \cap C_{b}^{1}(H)$ and

$$
K \varphi=L \varphi+\langle D \varphi, F\rangle
$$

for any $\varphi \in D(L) \cap C_{b}^{1}(H)$.

Proof Let $X(t, x)$ be the mild solution of $(1.1)$ and $Z(t, x)$ be the mild solution of (3.2), for any $\varphi \in D(L) \cap C_{b}^{1}(H)$, taking into account that

$$
\begin{aligned}
X(t, x)= & Z(t, x)+\int_{0}^{t} e^{(t-s) A} F(X(s, x)) d s \\
& +\int_{0}^{t} e^{(t-s) A}(G(X(s, x))-G(Z(s, x))) d W(s),
\end{aligned}
$$

by the Taylor formula we have $\mathbb{P}$-a.s.

$$
\begin{aligned}
\varphi(Z(t, x))= & \varphi(X(t, x))+\varphi(Z(t, x))-\varphi(X(t, x)) \\
= & \varphi(X(t, x))-\int_{0}^{1}\left\langle D \varphi(\xi Z(t, x)+(1-\xi) X(t, x)), \int_{0}^{t} e^{(t-s) A} F(X(s, x)) d s\right. \\
& \left.+\int_{0}^{t} e^{(t-s) A}(G(X(s, x))-G(Z(s, x))) d W(s)\right\rangle d \xi .
\end{aligned}
$$


Then we get

$$
\begin{aligned}
R_{t} \varphi(x)-\varphi(x)= & \mathbb{E}[\varphi(Z(t, x))]-\varphi(x) \\
= & P_{t} \varphi(x)-\varphi(x)-\mathbb{E}\left[\int_{0}^{1}\langle D \varphi(\xi Z(t, x)+(1-\xi) X(t, x)),\right. \\
& \left.\left.\int_{0}^{t} e^{(t-s) A} F(X(s, x)) d s\right\rangle d \xi\right]
\end{aligned}
$$

for any $x \in H$, with the help of Luigi Manca's result (see [3], Theorem 4.1), we have

$$
\begin{aligned}
& \lim _{t \rightarrow 0^{+}} \frac{1}{t} \mathbb{E}\left[\int_{0}^{1}\left\langle D \varphi(\xi Z(t, x)+(1-\xi) X(t, x)), \int_{0}^{t} e^{(t-s) A} F(X(s, x)) d s\right\rangle d \xi\right] \\
& \quad=\langle D \varphi(x), F(x)\rangle,
\end{aligned}
$$

so

$$
\lim _{t \rightarrow 0+} \frac{P_{t} \varphi(x)-\varphi(x)}{t}=L \varphi(x)+\langle D \varphi(x), F(x)\rangle .
$$

As is easily seen, $(L \varphi(x)+\langle D \varphi(x), F(x)\rangle) \in C_{b, 1}(H)$. Moreover,

$$
\begin{aligned}
\left|\frac{P_{t} \varphi(x)-\varphi(x)}{t}\right| \leq & \left|\frac{R_{t} \varphi(x)-\varphi(x)}{t}\right|+\frac{1}{t} \mathbb{E}\left[\mid \int_{0}^{1}\langle D \varphi(\xi Z(t, x)+(1-\xi) X(t, x)),\right. \\
& \leq\left|\frac{R_{t} \varphi(x)-\varphi(x)}{t}\right|+\|D \varphi\|_{0} \cdot \frac{1}{t} \mathbb{E}\left[\int_{0}^{t}\left|e^{(t-s) A} F(X(s, x))\right| d s\right],
\end{aligned}
$$

taking into account hypothesis $\left(\mathrm{H}_{3}\right)$ and (2.5), it yields

$$
\begin{aligned}
\left|\frac{P_{t} \varphi(x)-\varphi(x)}{t}\right| & \leq\left|\frac{R_{t} \varphi(x)-\varphi(x)}{t}\right|+\|D \varphi\|_{0} \cdot \frac{1}{t} \int_{0}^{t} \kappa(t-s)(1+\mathbb{E}[|X(s, x)|]) d s \\
& \leq\left|\frac{R_{t} \varphi(x)-\varphi(x)}{t}\right|+c\|D \varphi\|_{0}(1+|x|) \cdot \frac{1}{t} \int_{0}^{t} \kappa(s) d s,
\end{aligned}
$$

and due to $\kappa(t) \leq M_{1} e^{\omega_{1} t}$, for any $T>0$, there exists a constant $c_{1}>0$ such that

$$
\frac{1}{t} \int_{0}^{t} \kappa(s) d s \leq \frac{M_{1}\left(e^{\omega_{1} t}-1\right)}{\omega_{1} t} \leq c_{1}, \quad t \in(0, T] .
$$

This implies

$$
\sup _{t \in(0,1]}\left\|\frac{P_{t} \varphi(x)-\varphi(x)}{t}\right\|_{0,1}<\infty
$$

hence, $\varphi \in D(K) \cap C_{b}^{1}(H)$ and $K \varphi=L \varphi+\langle D \varphi, F\rangle$.

In the same way, for any $\varphi \in D(K) \cap C_{b}^{1}(H)$, we can prove that $\varphi \in D(L) \cap C_{b}^{1}(H)$, so this completes the proof. 
By Proposition 3.3 and Theorem 3.4 we have the following.

Corollary $3.5(K, D(K))$ is an extension of $K_{0}$ and for any $\varphi \in \mathscr{E}(A)$ we have $\varphi \in D(K)$ and $K \varphi=K_{0} \varphi$.

\subsection{The case $F \in C_{b}^{2}(H ; H)$}

The following lemma is proved in [10, Chapter 7].

Lemma 3.6 Let us assume Hypothesis 2.1 and that $F \in C_{b}^{2}(H ; H), G \in C_{b}^{2}(H ; L(H))$. Then the semigroup $\left\{P_{t}\right\}_{t \geq 0}$ defined in (1.5) maps $C_{b}^{1}(H)$ into $C_{b}^{1}(H)$, and for any $f \in C_{b}^{1}(H), h \in H$ we have

$$
\left\langle D P_{t} f(x), h\right\rangle=\mathbb{E}\left[\left\langle D f(X(t, x)), \eta^{h}(t, x)\right\rangle\right]
$$

where $\eta^{h}(t, x)$ is the mild solution of the equation in $H$

$$
\left\{\begin{aligned}
d \eta^{h}(t, x)= & \left(A \eta^{h}(t, x)+D F(X(t, x)) \cdot \eta^{h}(t, x)\right) d t \\
& +D G(X(t, x)) \cdot \eta^{h}(t, x) d W(t), \quad t>0, \\
\eta^{h}(0, x)= & h .
\end{aligned}\right.
$$

Proposition 3.7 Under the conditions of Lemma 3.6, we assume that the function $\kappa$ in hypothesis $\left(\mathrm{H}_{3}\right)$ satisfies $\kappa(t) \leq M_{1} e^{\omega_{1} t}, t \geq 0$, for two constants $M_{1}>0$ and $\omega_{1} \in \mathbb{R}$, let $(K, D(K))$ be the infinitesimal generator of $\left\{P_{t}\right\}_{t \geq 0}$. Then there exist two constants $\lambda_{0}>0$ and $\bar{M}>0$ such that for any $\lambda>\lambda_{0}$, the resolvent $R(\lambda, K)$ of $K$ at $\lambda$ maps $C_{b}^{1}(H)$ into $C_{b}^{1}(H)$ and we have

$$
\|D R(\lambda, K) f\|_{C_{b}(H ; H)} \leq \frac{\bar{M}\|D f\|_{C_{b}(H ; H)}}{\lambda-\lambda_{0}}, \quad f \in C_{b}^{1}(H)
$$

Proof Let $f \in C_{b}^{1}(H)$, for any $t \geq 0, P_{t} f \in C_{b}^{1}(H)$ and for any $x, h \in H$ we have

$$
\left\langle D P_{t} f(x), h\right\rangle=\mathbb{E}\left[\left\langle D f(X(t, x)), \eta^{h}(t, x)\right\rangle\right]
$$

where $\eta^{h}(t, x)$ is the mild solution of (3.19), that is,

$$
\begin{aligned}
\eta^{h}(t, x)= & e^{t A} h+\int_{0}^{t} e^{(t-s) A} D F(X(s, x)) \eta^{h}(s, x) d s \\
& +\int_{0}^{t} e^{(t-s) A} D G(X(s, x)) \eta^{h}(s, x) d W(s),
\end{aligned}
$$

then

$$
\begin{aligned}
\mathbb{E}\left[\left|\eta^{h}(t, x)\right|^{2}\right] \leq & 3\left(\left|e^{t A} h\right|^{2}+\mathbb{E}\left[\left|\int_{0}^{t} e^{(t-s) A} D F(X(s, x)) \eta^{h}(s, x) d s\right|^{2}\right]\right. \\
& \left.+\mathbb{E}\left[\left|\int_{0}^{t} e^{(t-s) A} D G(X(s, x)) \eta^{h}(s, x) d W(s)\right|^{2}\right]\right),
\end{aligned}
$$


applying the Minkowski inequality and the generalization of maximal inequality of martingales to stochastic convolution, see [6, Lemma 7.7 and Proposition 7.8], it follows that

$$
\begin{aligned}
\mathbb{E}\left[\left|\eta^{h}(t, x)\right|^{2}\right] \leq & 3\left(\left|e^{t A} h\right|^{2}+\mathbb{E}\left[\left(\int_{0}^{t}\left|e^{(t-s) A} D F(X(s, x)) \eta^{h}(s, x)\right| d s\right)^{2}\right]\right. \\
& \left.+c \mathbb{E}\left[\int_{0}^{t}\left\|e^{(t-s) A} D G(X(s, x)) \eta^{h}(s, x)\right\|_{L_{2}(H)}^{2} d s\right]\right),
\end{aligned}
$$

where $c$ is a given positive constant. By Hypothesis 2.1 and $F \in C_{b}^{2}(H ; H), G \in C_{b}^{2}(H ; L(H))$, we have

$$
e^{t A} D F \in C_{b}(H ; L(H)), \quad e^{t A} D G \in C_{b}\left(H ; L\left(H ; L_{2}(H)\right)\right)
$$

and

$$
\left|e^{t A} D F(x) y\right| \leq \kappa(t)|y|, \quad\left\|e^{t A} D G(x) y\right\|_{L_{2}(H)} \leq \kappa(t)|y|
$$

for any $x, y \in H$, considering that $\kappa(t) \leq M_{1} e^{\omega_{1} t}$, we obtain

$$
\begin{aligned}
\mathbb{E}\left[\left|\eta^{h}(t, x)\right|^{2}\right] \leq & 3\left(M^{2} e^{2 \omega t}|h|^{2}+\mathbb{E}\left[\left(\int_{0}^{t} \kappa(t-s)\left|\eta^{h}(s, x)\right| d s\right)^{2}\right]\right. \\
& \left.+c \mathbb{E}\left[\int_{0}^{t} \kappa^{2}(t-s)\left|\eta^{h}(s, x)\right|^{2} d s\right]\right) \\
\leq & 3\left(M^{2} e^{2 \omega t}|h|^{2}+M_{1}^{2} \mathbb{E}\left[\left(\int_{0}^{t} e^{\omega_{1}(t-s)}\left|\eta^{h}(s, x)\right| d s\right)^{2}\right]\right. \\
& \left.+c M_{1}^{2} \int_{0}^{t} e^{2 \omega_{1}(t-s)} \mathbb{E}\left[\left|\eta^{h}(s, x)\right|^{2}\right] d s\right) .
\end{aligned}
$$

By the Hölder inequality, one can obtain

$$
\begin{aligned}
\mathbb{E}\left[\left(\int_{0}^{t} e^{\omega_{1}(t-s)}\left|\eta^{h}(s, x)\right| d s\right)^{2}\right] & \leq \mathbb{E}\left[\left(\int_{0}^{t} e^{-(t-s)} d s\right)\left(\int_{0}^{t} e^{\left(2 \omega_{1}+1\right)(t-s)}\left|\eta^{h}(s, x)\right|^{2} d s\right)\right] \\
& \leq \int_{0}^{t} e^{\left(2 \omega_{1}+1\right)(t-s)} \mathbb{E}\left[\left|\eta^{h}(s, x)\right|^{2}\right] d s,
\end{aligned}
$$

then

$$
\begin{aligned}
\mathbb{E}\left[\left|\eta^{h}(t, x)\right|^{2}\right] \leq & 3\left(M^{2} e^{2 \omega t}|h|^{2}+M_{1}^{2} \int_{0}^{t} e^{\left(2 \omega_{1}+1\right)(t-s)} \mathbb{E}\left[\left|\eta^{h}(s, x)\right|^{2}\right] d s\right. \\
& \left.+c M_{1}^{2} \int_{0}^{t} e^{2 \omega_{1}(t-s)} \mathbb{E}\left[\left|\eta^{h}(s, x)\right|^{2}\right] d s\right) .
\end{aligned}
$$

Let $\bar{\omega}=\max \left\{\omega, \omega_{1}+\frac{1}{2}, 0\right\}$, multiplying (3.21) by $e^{-2 \bar{\omega} t}$ and taking into account $e^{2 \omega_{1}(t-s)} \leq$ $e^{\left(2 \omega_{1}+1\right)(t-s)} \leq e^{2 \bar{\omega}(t-s)}$, for $t>s$, yields

$$
e^{-2 \bar{\omega} t} \mathbb{E}\left[\left|\eta^{h}(t, x)\right|^{2}\right] \leq 3 M^{2}|h|^{2}+3 M_{1}^{2}(1+c) \int_{0}^{t} e^{-2 \bar{\omega} s} \mathbb{E}\left[\left|\eta^{h}(s, x)\right|^{2}\right] d s .
$$


Now from the Gronwall inequality it follows that

$$
\mathbb{E}\left[\left|\eta^{h}(t, x)\right|^{2}\right] \leq \bar{M}^{2} e^{2 \lambda_{0} t}|h|^{2},
$$

where the constants $\bar{M}=\sqrt{3} M, \lambda_{0}=\frac{3 M_{1}^{2}(1+c)}{2}+\bar{\omega}$, thus

$$
\mathbb{E}\left[\left|\eta^{h}(t, x)\right|\right] \leq \bar{M} e^{\lambda_{0} t}|h|
$$

By (iii) of Lemma 2.5, we have

$$
\begin{aligned}
|\langle D R(\lambda, K) f(x), h\rangle| & =\left|\int_{0}^{\infty} e^{-\lambda t} \mathbb{E}\left[\left\langle D f(X(t, x)), \eta^{h}(t, x)\right\rangle\right] d t\right| \\
& \leq \bar{M}\|D f\|_{C_{b}(H ; H)} \int_{0}^{\infty} e^{-\lambda t} e^{\lambda_{0} t}|h| d t \\
& =\frac{\bar{M}\|D f\|_{C_{b}(H ; H)}}{\lambda-\lambda_{0}}|h|
\end{aligned}
$$

for any $h \in H$. Therefore, (3.16) follows.

\subsection{The general case and the proof of Theorem 3.1}

From the above argument it remains to prove that $\mathscr{E}_{A}(H)$ is a $\pi$-core for $(K, D(K))$ for the proof of Theorem 3.1. For this we introduce the following approximation result (see [16]).

We take a sequence of nonnegative twice differentiable functions $\left\{\rho_{n}\right\}_{n \in \mathbb{N}}$ such that

$$
\operatorname{supp}\left(\rho_{n}\right) \subseteq\left\{\xi \in \mathbb{R}^{n},|\xi|_{\mathbb{R}^{n}} \leq \frac{1}{n}\right\} \quad \text { and } \quad \int_{\mathbb{R}^{n}} \rho_{n}(\xi) d \xi=1
$$

Let $Q_{n}$ be the orthonormal projection of $H$ onto $\operatorname{span}\left\{e_{1}, \ldots, e_{n}\right\},\left\{e_{n}\right\}_{n \in \mathbb{N}}$ be the orthonormal basis in $H$. We will identify $\mathbb{R}^{n}$ with $\operatorname{span}\left\{e_{1}, \ldots, e_{n}\right\}$, the mappings $F_{n}: H \rightarrow H$ and $G_{n}: H \rightarrow L(H)$ are defined by

$$
\begin{aligned}
& F_{n}(x)=\int_{\mathbb{R}^{n}} \rho_{n}\left(\xi-Q_{n} x\right) F\left(\sum_{i=1}^{n} \xi_{i} e_{i}\right) d \xi, \\
& G_{n}(x)=\int_{\mathbb{R}^{n}} \rho_{n}\left(\xi-Q_{n} x\right) G\left(\sum_{i=1}^{n} \xi_{i} e_{i}\right) d \xi .
\end{aligned}
$$

It is easy to check that $F_{n} \in C_{b}^{2}(H ; H), G_{n} \in C_{b}^{2}(H ; L(H))$ for any $n \in \mathbb{N}$. Moreover, $F_{n}(x) \rightarrow$ $F(x), G_{n}(x) \rightarrow G(x)$ as $n \rightarrow \infty$ for all $x \in H$, and $F_{n}, G_{n}, n \in \mathbb{N}$ satisfy $\left(\mathrm{H}_{1}\right)-\left(\mathrm{H}_{3}\right)$ in Hypothesis 2.1.

Let $P_{t}^{n}$ be the semigroup

$$
P_{t}^{n} \varphi(x)=\mathbb{E}\left[\varphi\left(X^{n}(t, x)\right)\right], \quad \varphi \in C_{b, 1}(H),
$$

where $X^{n}(t, x)$ is the mild solution of (1.1) with $F_{n}, G_{n}$ replacing $F, G$. Clearly, we have

$$
\lim _{n \rightarrow \infty} \mathbb{E}\left[\left|X^{n}(t, x)-X(t, x)\right|^{2}\right]=0, \quad t \geq 0, x \in H
$$


and for any $T>0$, there exists a constant $c>0$ such that

$$
\sup _{t \in[0, T]} \mathbb{E}\left[\left|X^{n}(t, x)\right|^{2}\right] \leq c\left(1+|x|^{2}\right), \quad x \in H .
$$

This implies

$$
\lim _{n \rightarrow \infty} \frac{P_{t}^{n} \varphi}{1+|\cdot|} \stackrel{\pi}{=} \frac{P_{t} \varphi}{1+|\cdot|}
$$

for any $t \geq 0, \varphi \in C_{b, 1}(H)$. We denote $\left(K_{n}, D\left(K_{n}\right)\right)$ the infinitesimal generator of the semigroup $P_{t}^{n}$ in $C_{b, 1}(H)$, defined as in (1.6) with $K_{n}, P_{t}^{n}$ replacing $K, P_{t}$. Also all the statements of Lemmas 2.4 and 2.5 hold for $P_{t}^{n}$ and $\left(K_{n}, D\left(K_{n}\right)\right)$. Combining (3.33), it is straightforward to see that the resolvent of $\left(K_{n}, D\left(K_{n}\right)\right)$ satisfy

$$
\lim _{n \rightarrow \infty} \frac{R\left(\lambda, K_{n}\right) \varphi}{1+|\cdot|} \stackrel{\pi}{=} \frac{R(\lambda, K) \varphi}{1+|\cdot|}
$$

for any $\varphi \in C_{b, 1}(H), \lambda>\omega_{0}$.

Proposition 3.8 The set $\mathscr{E}_{A}(H)$ is a $\pi$-core for $(K, D(K))$, and for any $\varphi \in D(K)$ there exist $m \in \mathbb{N}$ and an $m$-indexed sequence $\left\{\varphi_{n_{1}, \ldots, n_{m}}\right\}_{n_{1}, \ldots, n_{m} \in \mathbb{N}} \subset \mathscr{E}_{A}(H)$ such that

$$
\begin{aligned}
& \lim _{n_{1} \rightarrow \infty} \cdots \lim _{n_{m} \rightarrow \infty} \frac{\varphi_{n_{1}, \ldots, n_{m}}}{1+|\cdot|} \stackrel{\pi}{=} \frac{\varphi}{1+|\cdot|}, \\
& \lim _{n_{1} \rightarrow \infty} \cdots \lim _{n_{m} \rightarrow \infty} \frac{K_{0} \varphi_{n_{1}, \ldots, n_{m}}}{1+|\cdot|} \stackrel{\frac{\pi}{=}}{\frac{K \varphi}{1+|\cdot|}} .
\end{aligned}
$$

Proof The proof goes along the same lines as that of Lemma 4.6 in [3], with some important changes. Namely, instead of Propositions 4.3 and 4.5 in [3], we have to use the modifications which correspond to Propositions 3.3 and 3.7 above.

\section{Fokker-Planck equation for Kolmogorov operator}

This section is devoted to studying the following Fokker-Planck equation for the Kolmogorov operator $K_{0}$ :

$$
\left\{\begin{array}{l}
\frac{d}{d t} \int_{H} \varphi(x) \mu_{t}(d x)=\int_{H} K_{0} \varphi(x) \mu_{t}(d x), \quad t \geq 0, \varphi \in \mathscr{E}_{A}(H) \\
\mu_{0}=\mu \in \mathcal{M}_{1}(H)
\end{array}\right.
$$

where the Kolmogorov operator $K_{0}$ is defined by (1.7).

To give a precise meaning of this problem, we introduce the notion of solution of (4.1).

Definition 4.1 Given $\mu \in \mathcal{M}_{1}(H)$, we say that a family of measures $\left\{\mu_{t}\right\}_{t \geq 0}$ is a solution of the Fokker-Planck equation (4.1) if the following are fulfilled:

(i) the total variation of the measure $\mu_{t}$ satisfies

$$
\int_{0}^{T} \int_{H}(1+|x|)\left|\mu_{t}\right|(d x) d t<\infty, \quad \forall T>0
$$


(ii) for any $\varphi \in \mathscr{E}_{A}(H)$ and any $t \geq 0$, it holds

$$
\int_{H} \varphi(x) \mu_{t}(d x)-\int_{H} \varphi(x) \mu(d x)=\int_{0}^{t}\left(\int_{H} K_{0} \varphi(x) \mu_{s}(d x)\right) d s
$$

From Theorem 3.1 we know the relationships between the Kolmogorov operator $K_{0}$ and the infinitesimal generator $(K, D(K))$ of the transition semigroup $\left\{P_{t}\right\}_{t \geq 0}$, defined by (1.6). Then we firstly study the measure value equation

$$
\left\{\begin{array}{l}
\frac{d}{d t} \int_{H} \varphi(x) \mu_{t}(d x)=\int_{H} K \varphi(x) \mu_{t}(d x), \quad t \geq 0, \varphi \in D(K), \\
\mu_{0}=\mu \in \mathcal{M}_{1}(H) .
\end{array}\right.
$$

Theorem 4.2 Assume that the conditions of Theorem 3.1 hold, let $\left\{P_{t}\right\}_{t \geq 0}$ be the semigroup associated to the SPDE (1.1) defined by $(1.5)$ and $(K, D(K))$ be its infinitesimal generator defined by (1.6). Then the formula

$$
\left\langle\varphi, P_{t}^{*} f\right\rangle_{\sigma\left(C_{b, 1}(H), C_{b, 1}(H)^{*}\right)}=\left\langle P_{t} \varphi, f\right\rangle_{\sigma\left(C_{b, 1}(H), C_{b, 1}(H)^{*}\right)}
$$

defines a semigroup $\left\{P_{t}^{*}\right\}_{t \geq 0}$ of linear and continuous operators on $C_{b, 1}(H)^{*}$ that maps $\mathcal{M}_{1}(H)$ into $\mathcal{M}_{1}(H)$. Moreover, for any $\mu \in \mathcal{M}_{1}(H)$ there exist a unique family of measures $\left\{\mu_{t}\right\}_{t \geq 0} \subset \mathcal{M}_{1}(H)$ such that

$$
\int_{0}^{T} \int_{H}(1+|x|)\left|\mu_{t}\right|(d x) d t<\infty, \quad \forall T>0
$$

and

$$
\int_{H} \varphi(x) \mu_{t}(d x)-\int_{H} \varphi(x) \mu(d x)=\int_{0}^{t}\left(\int_{H} K \varphi(x) \mu_{s}(d x)\right) d s
$$

for any $t \geq 0, \varphi \in D(K)$. Finally, the solution of (4.4) is given by $P_{t}^{*} \mu, t \geq 0$.

Proof In order to proof our result, we can follow almost the same arguments as in the proof of Theorem 1.2 in [5] by Luigi Manca. We omit the details here.

As a consequence we get the second main result.

Theorem 4.3 Assume that the conditions of Theorem 3.1 hold, for any $\mu \in \mathcal{M}_{1}(H)$ there exists a unique solution of measures $\left\{\mu_{t}\right\}_{t \geq 0} \subset \mathcal{M}_{1}(H)$ of (4.1), and this solution is given by $P_{t}^{*} \mu$.

Proof Let $(K, D(K))$ be the infinitesimal generator defined by (1.6), by Theorem 3.1 we find that $\mathscr{E}_{A}(H)$ is a $\pi$-core for $(K, D(K))$, and that $K \varphi=K_{0} \varphi$, for any $\varphi \in \mathscr{E}_{A}(H)$. So combining Theorem 4.2 it is easy to show that $\left\{P_{t}^{*} \mu\right\}_{t \geq 0}$ is a solution of the Fokker-Planck equation (4.1) for any $\mu \in \mathcal{M}_{1}(H)$.

To prove the uniqueness of the solution, we assume that $\left\{\mu_{t}\right\}_{t \geq 0}$ is a solution of (4.1). For any $t \geq 0$ and $\varphi \in D(K)$, there exists a sequence $\left(\varphi_{n}\right)_{n \in \mathbb{N}} \subset \mathscr{E}_{A}(H)$ (for simplicity we 
assume that this sequence has only one index) such that

$$
\lim _{n \rightarrow \infty} \frac{\varphi_{n}}{1+|\cdot|} \stackrel{\pi}{=} \frac{\varphi}{1+|\cdot|}, \quad \lim _{n \rightarrow \infty} \frac{K_{0} \varphi_{n}}{1+|\cdot|} \stackrel{\pi}{=} \frac{K \varphi}{1+|\cdot|},
$$

and we have

$$
\begin{aligned}
\int_{H} \varphi(x) \mu_{t}(d x)-\int_{H} \varphi(x) \mu(d x) & =\lim _{n \rightarrow \infty}\left(\int_{H} \varphi_{n}(x) \mu_{t}(d x)-\int_{H} \varphi_{n}(x) \mu(d x)\right) \\
& =\lim _{n \rightarrow \infty} \int_{0}^{t}\left(\int_{H} K_{0} \varphi_{n}(x) \mu_{s}(d x)\right) d s .
\end{aligned}
$$

Now observe that $\sup _{n \in \mathbb{N}}\left|K_{0} \varphi_{n}\right| \leq c(1+|x|)$ for some $c>0$ and $\mu_{s} \in \mathcal{M}_{1}(H)$ for any $s \geq 0$,

so

$$
\lim _{n \rightarrow \infty} \int_{H} K_{0} \varphi_{n}(x) \mu_{s}(d x)=\int_{H} K \varphi(x) \mu_{s}(d x)
$$

and

$$
\sup _{n \in \mathbb{N}}\left|\int_{H} K_{0} \varphi_{n}(x) \mu_{s}(d x)\right| \leq c \int_{H}(1+|x|)\left|\mu_{s}\right|(d x) .
$$

Taking into account (4.2) and applying the dominated convergence theorem, it yields

$$
\lim _{n \rightarrow \infty} \int_{0}^{t}\left(\int_{H} K_{0} \varphi_{n}(x) \mu_{s}(d x)\right) d s=\int_{0}^{t}\left(\int_{H} K \varphi(x) \mu_{s}(d x)\right) d s
$$

this implies that $\left\{\mu_{t}\right\}_{t \geq 0}$ is a solution of the measure equation (4.4). On the other hand such a solution is unique and is given by $\left\{P_{t}^{*} \mu\right\}_{t \geq 0}$ by Theorem 4.2 , that is, for any $\varphi \in \mathscr{E}_{A}(H)$ we have

$$
\int_{H} \varphi(x) P_{t}^{*} \mu(d x)=\int_{H} \varphi(x) \mu_{t}(d x)
$$

By Proposition 3.2, (4.11) still holds for any $\varphi \in C_{b}(H)$, this implies $\mu_{t}=P_{t}^{*} \mu, \forall t \geq 0$. This concludes the proof.

\section{Competing interests}

The authors declare that they have no competing interests.

Authors' contributions

All authors contributed equally to the writing of this paper. All authors read and approved the final manuscript.

\section{Acknowledgements}

This work was partially supported by NNSF of China (Grant No. 11171122).

Received: 1 March 2014 Accepted: 28 July 2014 Published: 15 August 2014

\section{References}

1. Cerrai, S: Stochastic reaction-diffusion systems with multiplicative noise and non-Lipschitz reaction term. Probab. Theory Relat. Fields 125, 271-304 (2003)

2. Stannat, W: Stochastic partial differential equations: Kolmogorov operators and invariant measures. Jahresber. Dtsch. Math.-Ver. 113, 81-109 (2011) 
3. Manca, L: Fokker-Planck equation for Kolmogorov operators with unbounded coefficients. Stoch. Anal. Appl. 27, 747-769 (2009)

4. Goldys, B, Kocan, M: Diffusion semigroups in spaces of continuous functions with mixed topology. J. Differ. Equ. 173, 17-39 (2001)

5. Manca, L: Kolmogorov equations for measures. J. Evol. Equ. 8, 231-262 (2008)

6. Da Prato, G, Zabczyk, J: Stochastic Equations in Infinite Dimensions. Encyclopedia of Mathematics and Its Applications, vol. 44. Cambridge University Press, Cambridge (1992)

7. Da Prato, G: Kolmogorov Equations for Stochastic PDEs. Advanced Courses in Mathematics - CRM Barcelona. Birkhäuser, Basel (2004)

8. Da Prato, $G$, Debussche, A: $m$-Dissipativity of Kolmogorov operators corresponding to Burgers equations with space-time white noise. Potential Anal. 26, 31-55 (2007)

9. Da Prato, G, Zabczyk, J: Ergodicity for Infinite Dimensional Systems. London Mathematical Society Lecture Notes, vol. 293. Cambridge University Press, Cambridge (1996)

10. Da Prato, G, Zabczyk, J: Second Order Partial Differential Equations in Hilbert Space. London Mathematical Society Lecture Notes, vol. 283. Cambridge University Press, Cambridge (2002)

11. Manca, L: On a class of stochastic semilinear PDEs. Stoch. Anal. Appl. 24, 399-426 (2006)

12. Bogachev, VI, Da Prato, G, Röckner, M: Fokker-Planck equations and maximal dissipativity for Kolmogorov operators with time dependent singular drifts in Hilbert spaces. J. Funct. Anal. 256, 1269-1298 (2009)

13. Bogachev, VI, Da Prato, G, Röckner, M: Existence results for Fokker-Planck equations in Hilbert spaces. In: Dalang, R, Dozzi, M, Russo, F (eds.) Seminar on Stochastic Analysis, Random Fields and Applications VI (Centro Stefano Franscini, Ascona, May 2008). Progress in Probability, vol. 63, pp. 23-35. Springer, Basel (2011)

14. Bogachev, VI, Röckner, M: Elliptic equations for measures on infinite dimensional spaces and applications. Probab. Theory Relat. Fields 120, 445-496 (2001)

15. Priola, E: On a class of Markov type semigroups in spaces of uniformly continuous and bounded functions. Stud. Math. 136, 271-295 (1999)

16. Peszat, S, Zabczyk, J: Strong Feller property and irreducibility for diffusions on Hilbert spaces. Ann. Probab. 23, 157-172 (1995)

doi:10.1186/1687-1847-2014-222

Cite this article as: Shi and Liu: Fokker-Planck equation for Kolmogorov operators associated to stochastic PDE with multiplicative noise. Advances in Difference Equations 2014 2014:222.

\section{Submit your manuscript to a SpringerOpen ${ }^{\circ}$ journal and benefit from:}

- Convenient online submission

- Rigorous peer review

- Immediate publication on acceptance

- Open access: articles freely available online

- High visibility within the field

- Retaining the copyright to your article 